\title{
EXAMPLES OF UNGRADABLE ALGEBRAS
}

\author{
TH. BELZNER, W. D. BURGESS, K. R. FULLER, AND R. SCHULZ
}

(Communicated by Maurice Auslander)

\begin{abstract}
Examples are presented of finite-dimensional algebras that admit no positive grading (that is, a nontrivial grading indexed by the natural numbers). Some of these examples have finite global dimension (they are even quasihereditary), and yield a negative answer to a question of Anick and Green.
\end{abstract}

A finite-dimensional algebra $A$ over a field $K$ has a positive semisimple grading in case there is a $K$-decomposition

$$
A=\bigoplus_{n \geq 0} A_{n}
$$

such that $A_{m} A_{n} \subseteq A_{m+n}$ for all nonnegative integers $m$ and $n$, and the radical of $A$ is

$$
J=\bigoplus_{n>0} A_{n}
$$

In this case the initial subring $A_{0} \cong A / J$. (In [1] such an algebra is called nontrivially $\mathbf{N}$-gradable.)

It is easy to see that any split finite-dimensional $K$-algebra $A$ with radical $J=J(A)$ such that $J^{3}=0$ has a positive semisimple grading. Indeed, then $A$ contains a subalgebra $A_{0}$ (isomorphic to a direct sum of matrix rings over $K$ ) such that ${ }_{K} A=A_{0} \oplus J$ and $A_{0} \otimes_{K} A_{0}^{o p}$ is semisimple, so that $J=A_{1} \oplus J^{2}$ as an $A_{0}-A_{0}$ bimodule; the grading is $A=A_{0} \oplus A_{1} \oplus A_{2}$ with $A_{2}=J^{2}$. In particular, any split algebra of dimension four over $K$ has a positive semisimple grading because it must have $J^{3}=0$ or be uniserial.

With these observations in mind we present a split $K$-algebra with

$$
\operatorname{dim}\left({ }_{K} A\right)=5 \text { and } J^{4}=0
$$

that admits no positive semisimple grading.

1. Example. In the free associative algebra $K\langle X, Y\rangle$ over a field $K$, let $I$ be the ideal generated by the elements $X^{3}, X Y, Y X^{2}, X^{2}-Y^{3}$, and $Y X-Y^{3}$,

Received by the editors June 19, 1990.

1980 Mathematics Subject Classification (1985 Revision). Primary 16A03, 16A46.

Key words and phrases. Graded algebras, ungradable algebras, finite global dimension.

The second author's research was supported by grant A7539 of the NSERC.

The third author's research was supported by a grant from the National Security Agency. 
and let $A=K\langle X, Y\rangle / I$. Then $A$ is a local $Q F$ algebra that admits no positive semisimple grading.

Proof. Let $x$ and $y$ denote the residue classes of $X$ and $Y$, respectively. Then the elements $1, x, y, y^{2}$, and $y^{3}$ form a $K$-basis of $A$, and we have $x^{2}=y x=y^{3}, x y=0$, and $y^{4}=0$. Letting $J=J(A)$, direct calculations show that the left and right lower Loewy series of $A$ coincide (we denote them by $S=\operatorname{Soc}(R), S_{2}=\operatorname{Soc}_{2}(R)$, etc.) and that

$$
\begin{gathered}
J=S_{3}=K x \oplus K y \oplus K y^{2} \oplus K y^{3} \\
S_{2}=K x \oplus K y^{2} \oplus K y^{3}=\left\{a \in A: a^{3}=0\right\} \\
J^{2}=K y^{2} \oplus K y^{3}=\left\{a \in A: a^{2}=0\right\} \\
J^{3}=S=K y^{3} .
\end{gathered}
$$

It follows from (4) that $A$ is $Q F$. Considering the $K$-dimension and the Loewy length of $A$, we see that a positive semisimple grading of $A$ must have either four or five nonzero terms. We shall show that neither is possible.

Suppose that there is a grading of the form

$$
A=A_{0} \oplus A_{i} \oplus A_{j} \oplus A_{k} \oplus A_{l}
$$

with each $\operatorname{dim} A_{n}=1$ and $0<i<j<k<l$. Then clearly $A_{l}=J^{3} \neq 0$, so one of $A_{i}^{2}, A_{i} A_{j}, A_{j} A_{i}$, or $A_{j}^{2}$ is not contained in $A_{l}$. If $A_{i}^{2} \nsubseteq A_{l}$, then $A_{i}^{2}=A_{j}$ or $A_{i}^{2}=A_{k}$. But if $A_{i}^{2}=A_{j}$ then $J^{2}=A_{j} \oplus A_{l}$ and by (3), $0 \neq A_{k}^{2}=A_{l}$ and by $(2), 0 \neq A_{i}^{3}=A_{l}$. This leads to the contradiction

$$
4 i=2 j<2 k=l=3 i \text {. }
$$

Thus all products $A_{m} A_{n}$ must be contained in $A_{k} \oplus A_{l}$, and so

$$
A_{k} \oplus A_{l}=J^{2} \text {. }
$$

Now, since $J^{3} \neq 0$, it follows that $A_{i} A_{k}=A_{l}$ or $A_{j} A_{k}=A_{l}$. The latter implies $A_{i} A_{k}=0$, so $A_{i} J^{2}=0$ and hence $A_{i} \subseteq S_{2}$ but $A_{i} \nsubseteq J^{2}$. Then by (3) $A_{i}^{2}=A_{l}$, which yields the contradiction $j+k=l=2 i$. Finally, suppose that $A_{i} A_{k}=A_{l}$, then $A_{j} A_{k}=0$ so $A_{j} J^{2}=0$ and $S_{2}=A_{j} \oplus A_{k} \oplus A_{l}$. Now by (2) and (3) there are $K$-generators $a$ for $A_{i}$ and $b$ for $A_{j}$ that are $K$-linear combinations of the form

$$
a=\kappa_{1} x+y+\kappa_{3} y^{2}+\kappa_{4} y^{3}
$$

and

$$
b=x+\lambda_{3} y^{2}+\lambda_{4} y^{3} .
$$

But since $A_{i} A_{j} \subseteq J S_{2} \subseteq A_{l}$ and $i+k=l, A_{i} A_{j}=0=A_{j} A_{i}$, so this implies

$$
\left(\kappa_{1}+1+\lambda_{3}\right) y^{3}=a b=0=b a=\left(\kappa_{1}+\lambda_{3}\right) y^{3} .
$$

Thus a positive semisimple grading with five nonzero terms cannot exist.

The remaining possibility is a positive semisimple grading of the form

$$
A=A_{0} \oplus A_{i} \oplus A_{j} \oplus A_{k}
$$


with each $A_{n} \neq 0$. Here we must have $0 \neq J^{3}=A_{i}^{3}$, which implies

$$
j=2 i \text { and } k=3 i \text {; }
$$

and consequently $A_{j}^{2}=0$. Thus by (3), $A_{j} \oplus A_{k}=J^{2}$ and if

$$
x=a_{i}+a_{j}+a_{k}
$$

with the $a_{n} \in A_{n}$ then $a_{i}$ must have no $y$-coordinate. But then by (2), $a_{i} J^{2}=$ $0=J^{2} a_{i}$, so

$$
y^{3}=x^{2}=a_{i}^{2} \in A_{k} \cap A_{j}=0 .
$$

This contradiction completes the proof.

In [2, Theorem 10.3] Auslander showed that if $A$ is a finite-dimensional $K$ algebra with radical $J$ and Loewy length $l$ then the algebra $\operatorname{End}\left({ }_{A} M\right)$, with $M=A / J \oplus \cdots \oplus A / J^{l-1} \oplus A$ has global dimension $\leq l$. This construction, together with the example above, yields a split finite-dimensional $K$-algebra of finite global dimension which admits no positive semisimple grading.

2. Proposition. Let $A$ be a finite-dimensional $K$-algebra, ${ }_{A} M$ be a finitely generated generator, and $B=\operatorname{End}\left({ }_{A} M\right)$. If $A$ admits no positive semisimple grading, then neither does $B$.

Proof. It follows from [7, Proposition 2.4] that the existence of a positive semisimple grading is a Morita invariant, so we may assume that ${ }_{A} M=N \oplus A$. Suppose that $B=\operatorname{End}\left({ }_{A} M\right)$ and there is such a grading

$$
B=\bigoplus_{n \geq 0} B_{n}
$$

Then according to [3, Proposition 9] $B_{0}$ contains a complete set of primitive idempotents for $B$, so there is an idempotent $e \in B_{0}$ such that

$$
e B e \cong \operatorname{End}\left({ }_{A} A\right) \cong A \text {. }
$$

This yields a positive semisimple grading

$$
A \cong e B e=\bigoplus_{n \geq 0} e B_{n} e .
$$

3. Remarks. (a) The fact is that the algebra $A$ of Example 1 does not admit any nontrivial integral grading. Indeed, if ${ }_{K} A=\bigoplus_{n \in \mathrm{Z}} A_{n}$ with $A_{j} A_{k} \subseteq A_{j+k}$ and $J_{0}=J\left(A_{0}\right)$, then $J=J_{0} \oplus\left(\bigoplus_{n \neq 0} A_{n}\right)$ and there are at most four nonzero terms with $n \neq 0$. If there were only three of these whose indices were not all of the same sign, or if there were two with positive indices and two with negative indices, then the cube of each term in $J=J_{0} \oplus\left(\bigoplus_{n \neq 0} A_{n}\right)$ would be zero, contrary to (2). A grading with nonzero terms $A=A_{i} \oplus A_{0} \oplus A_{j} \oplus A_{k} \oplus A_{l}$ and $i<0<j<k<l$ would have $J^{2}=A_{i} \oplus A_{l}$ by (3), and $J_{0}=0$, leading to $A_{i}=J^{3}=A_{l}$. Thus we see that in an integral grading of $A$ the indices of the nonzero terms must all be nonnegative (or what is the same, nonpositive). Thus, in view of Example 1, we are left with the possibility of $J_{0} \neq 0$ and one, two, or three nonzero terms with positive indices; and then since $A_{0}$ must be a proper factor ring of $A, J_{0}^{3}=0$. The cases of one or two nonzero terms can be eliminated using (2) as before. Finally, a grading $A=A_{0} \oplus A_{i} \oplus A_{j} \oplus A_{k}$ with 
$0<i<j<k$ and $J_{0} \neq 0$ would have $J_{0}^{2}=A_{j}^{3}=A_{k}^{2}=0$, so by (3) $A_{i}^{2}=A_{j}$ and $A_{i}^{3}=A_{k}$. This implies $j=2 i, k=3 i$, and so $A_{j}^{2} \subseteq A_{4 i}=0$. Thus by (3), we arrive at the contradiction $J_{0} \oplus A_{j} \oplus A_{k} \subseteq J^{2}$.

(b) Exercise 1 in $[8, \S 11.6]$ presents a four-dimensional commutative algebra $R$ over a field $F$ which is uniserial of composition length 2 such that $R / J(R)$ is not isomorphic to any subring of $R$ (and, so, $R$ is not split). If $R=\bigoplus_{n \in Z} R_{n}$ were a nontrivial grading then each $R_{i}, i \neq 0$, being nilpotent, is in $J(R)$. But $\operatorname{dim} J(R)=2$ and $R_{0} \neq R / J(R)$, so $R=R_{0} \oplus R_{i}, i \neq 0$ and $\operatorname{dim} R_{i}=1$. But then $R_{i}$ is an ideal and $R$ has no ideals of dimension one.

If $C=\operatorname{End}(R(R / J(R) \oplus R))$, then $C$ is of global dimension 2 and admits no nontrivial positive grading $C=C_{0} \oplus \cdots \oplus C_{m}$ with $C_{m} \neq 0$. Indeed, such a grading would yield $R \cong e C_{0} e \oplus e C_{m} e$ where $e$ is the projection on ${ }_{R} R$ in $C$, but $e C_{m} e \neq 0$ since $C_{m}$ is an ideal and $e C$ and $C e$ are $C$-faithful.

(c) It was shown in [6] that the endomorphism algebra $\operatorname{End}\left({ }_{A} M\right)$, with $M=A / J \oplus \cdots \oplus A / J^{l-1} \oplus A$, is in fact quasihereditary (see [4] or [5] for the definition). Hence there are even quasihereditary algebras with no positive semisimple gradings.

(d) A curious example of a split commutative algebra is obtained when

$$
A=K[X, Y] /\left(X^{4}-X^{2} Y+X Y^{2}, X^{4}-X^{3}, X^{5}, Y^{4}\right) \text {. }
$$

When $K$ is of characteristic not equal to $2, A$ can be graded. For example, $A_{0}=K \cdot 1, A_{3}=K \cdot\left(x-\frac{1}{2} y\right), A_{4}=K \cdot\left(-\frac{5}{4} x^{2}+y\right), A_{6}=K \cdot\left(x^{2}+\frac{1}{4} y^{2}-x y\right), A_{7}=$ $K \cdot\left(-\frac{5}{4} x^{3}-\frac{1}{2} y^{2}+x y+\frac{5}{8} x^{2} y\right), A_{8}=K \cdot\left(y^{2}-\frac{5}{2} x^{2} y\right), A_{9}=K \cdot\left(x^{3}-\frac{7}{8} x^{4}-\frac{3}{4} x^{2} y\right)$, $A_{11}=K \cdot\left(-\frac{3}{2} x^{4}+x y^{2}\right)$, and $A_{12}=K \cdot x^{4}$, where $x$ and $y$ are the images, respectively, of $X$ and $Y$ in $A$ and the $K$-basis $\left\{1, x, x^{2}, x^{3}, x^{4}, y, y^{2}, x y, x^{2} y\right\}$ is chosen. On the other hand it can be shown that if $K=\mathbf{Z} / 2 \mathbf{Z}, A$ has no nontrivial integral grading.

\section{REFERENCES}

1. D. J. Anick and E. L. Green, On the homology of quotients of path algebras, Comm. Algebra 15 (1987), 309-342.

2. M. Auslander, Representation theory of artin algebras II, Comm. Algebra 1 (1974), 177-268.

3. V. P. Camillo and K. R. Fuller, On graded rings with finiteness conditions, Proc. Amer. Math. Soc. 86 (1982), 1-5.

4. E. Kline, B. Parshall, and L. Scott, Finite dimensional algebras and highest weight categories, J. Reine Angew. Math. 391 (1988), 277-291.

5. V. Dlab and C. M. Ringel, Quasihereditary algebras, Illinois J. Math. 33 (1989), 280-291. 1988.

6. __ Every semiprimary ring is the endomorphism ring of a projective module over a quasihereditary ring, Proc. Amer. Math Soc. 107 (1989), 1-5.

7. R. Gordon and E. L. Green, Graded artin algebras, J. Algebra 76 (1982), 111-137.

8. R. S. Pierce, Associative algebras, Springer-Verlag, Berlin, Heidelberg, and New York, 1982.

Software, Design, and Management, Thomas-Dehler-Str. 27, 8000 München 83, GerMANY

Department of Mathematics, University of Ottawa, Ottawa, Canada K1N 6N5

Department of Mathematics, The University of Iowa, Iowa City, Iowa, 52242

National University of Singapore, Department of Mathematics, Lower Kent Ridge ROAD, SINGAPORE 0511 